\title{
The late-time development of the Richtmyer-Meshkov instability
}

\author{
J. K. Prasad, ${ }^{\text {a) }}$ A. Rasheed, S. Kumar, and B. Sturtevant \\ Graduate Aeronautical Laboratories, California Institute of Technology, Pasadena, California 91125
}

(Received 20 May 1999; accepted 3 April 2000)

\begin{abstract}
Measurements have been made of the growth by the Richtmyer-Meshkov instability of nominally single-scale perturbations on an air/sulfur hexafluoride $\left(\mathrm{SF}_{6}\right)$ interface in a large shock tube. An approximately sinusoidal shape is given to the interface by a wire mesh which supports a polymeric membrane separating the air from the $\mathrm{SF}_{6}$. A single shock wave incident on the interface induces motion by the baroclinic mechanism of vorticity generation. The visual thickness $\delta$ of the interface is measured from schlieren photographs obtained singly in each run and in high-speed motion pictures. Data are presented for $\delta$ at times considerably larger than previously reported, and they are tested for self-similarity including independence of initial conditions. Four different initial amplitude/wavelength combinations at one incident shock strength are used to determine the scaling of the data. It is found that the growth rate decreases rapidly with time, $d \delta / d t \propto t^{-p}$ (i.e., $\delta$ $\propto t^{1-p}$ ), where $0.67 \lesssim p \lesssim 0.74$ and that a small dependence on the initial wavelength $\lambda_{0}$ persists to large time. The larger value of the power law exponent agrees with the result of the late-time-decay similarity law of Huang and Leonard [Phys. Fluids 6, 3765-3775 (1994)]. The influence of the wire mesh and membrane on the mixing process is assessed. (c) 2000 American Institute of Physics.
\end{abstract} [S1070-6631(00)00907-7]

\section{INTRODUCTION}

When a shock wave encounters an interface between two fluids of different density it generates vorticity by the baroclinic mechanism in a process known as the RichtmyerMeshkov (RM) instability. ${ }^{1,2}$ Perturbations of the shape of the interface do not initially grow exponentially as in the Rayleigh-Taylor (RT) instability, but instead grow linearly in time. The resulting motions are themselves KelvinHelmholtz unstable, so the range of physical scales increases as vortex sheets roll up. Even in flows with initially twodimensional perturbations, three dimensionality develops, perhaps due to a mixing transition, ${ }^{3-5}$ and the flow becomes turbulent in the neighborhood of the interface. Whether all of these processes can develop before the motions decay by viscous dissipation depends on the magnitude of the Reynolds number based on the interface velocity and a typical spatial scale.

Research interest has focused on initially two- or threedimensional sinusoidal perturbations (the so-called "singlemode" case) as a test case for theories, and on small-scale random perturbations (the "multimode" case) for their relevance to applications. The "turbulence" generated by plane shocks is initially highly anisotropic, the vorticity lying primarily in the plane of the shock wave. In the multimode case, the baroclinically-produced vorticity can be thought of as a random array of parallel vortex rings.

It is usually assumed that after a long enough time, the turbulence in the turbulent mixing zone (TMZ) becomes isotropic. If, further, time is large enough that a large range of

\footnotetext{
a)Present address: Vikram Sarabhai Space Center, Trivandrum 695022, India.
}

scales has developed, it is thought that the flow becomes independent of the initial conditions and is controlled only by local, time-varying scales. The behavior is then selfsimilar and an appropriately defined thickness of the turbulent mixing zone (TMZ) grows as a power law of the time $t$ after shock excitation,

$$
\delta \propto t^{n} .
$$

In this paper, we define $\delta$ to be the half-width of the turbulent mixing zone (TMZ) including both large-scale and small-scale distortions. Haan ${ }^{6}$ discussed late-stage growth and suggested that for large enough time the amplitudes of initially single-scale and multiscale perturbations should be comparable, though the distribution of phases may not be. He proposed a model which exhibits small but finite dependence on initial conditions. Barenblatt ${ }^{7}$ used the Kolmogorov similarity hypothesis to show that the upper-limit of growth rate for a dissipationless fluid is $n=2 / 3$. Mikaelian ${ }^{8}$ used the method by which Richtmyer ${ }^{1}$ had treated the linear case to extrapolate the late-time constant-acceleration RayleighTaylor (RT) experimental results of Read ${ }^{9}$ to the impulsiveacceleration Richtmyer-Meshkov (RM) case, obtaining

$$
\delta=0.14 A U t,
$$

where $A=\left(\rho_{2}-\rho_{1}\right) /\left(\rho_{2}+\rho_{1}\right)$ is the Atwood number and $U$ is the velocity to which the interface is accelerated by the shock wave. This result was applied to a study of turbulent energy in the large-scale structures of the flow (greater than the dissipation scale), where the Barenblatt constraint $n$ $\leqslant 2 / 3$ need not apply. ${ }^{9}$

Alon et al..$^{10,11}$ analyzed the late-time growth of multimode fronts using a bubble merging and competition model. For $A \neq 1$, they argued that both crests and troughs should increase amplitude with $n \doteq 0.4$. The growth depends 
strongly on the initial conditions; amplitude is scaled by $\lambda_{0}$, where $\lambda_{0}$ is the initial wavelength, and time by $\lambda_{0} / u_{0}$, where

$$
u_{0}=k_{0} A U \eta_{0}
$$

is the growth rate predicted by the linear theory. ${ }^{1}$ Here $k_{0}$ $=2 \pi / \lambda_{0}$ is the wave number and $\eta_{0}$ is the initial halfamplitude of the perturbation. Zhang and Sohn ${ }^{12}$ developed a theory of nonlinear growth by extending the linear theory ${ }^{1}$ in a Taylor series in amplitude, enlarging the range of validity using a Padé approximation and requiring that the result conform to the linear stability theory [Eq. (3)] at small time. Their nonlinear theory agrees better with the shock tube experiments of Benjamin, Besnard, and Haas (unpublished, 1993) than does linear theory, suggesting that the experimental conditions were in the nonlinear regime. This is consistent with the observation of Benjamin ${ }^{13}$ that the interface was nonlinearly deformed, exhibiting bubbles and spikes, despite the fact that its thickness was growing linearly in time. The late-time overall growth rate varies as $1 / t$ for relatively large $A$, as do their predictions of the separate bubble and spike growth rates for all $A$, but the overall growth rate goes as $1 / t^{2}$ for small $A$. For the Atwood numbers of the present experiments, the prediction ${ }^{12}$ is $\delta \underset{\sim}{\propto} \ln t$. Sadot et al. ${ }^{14}$ gave a simple interpolation formula between small and large time behavior which also behaves as $\delta \propto \ln t$ for large time. Neither of these results is obtained from scaling arguments; rather they are inferred from power-series solutions and numerical models. Pham and Meiron ${ }^{15}$ simulated the late-time behavior of the multimode RM and found $n \approx 0.4$. We have not discussed recent work on the case $A=1$, because there the spikes show quite different scaling not relevant to the present experiments.

At very late times the turbulent motions are slow, the flow becomes viscous and the local Reynolds number,

$$
\operatorname{Re}=u^{\prime} \delta / \nu \sim \delta^{2} / \nu t
$$

may be constant or may even decrease with time. Here $u^{\prime}$ is the late-time turbulent velocity scale which we assume to be of order $u^{\prime} \sim d \delta / d t \sim \delta / t$. The behavior at these times has recently been studied by Huang and Leonard, ${ }^{16}$ who propose a physically-reasonable late-time similarity that yields a power law decay for the turbulent energy. Using the hypothesis of Saffman, ${ }^{17}$ that the integral moments of the vorticity distribution in the large scales of turbulence are bounded, an invariant of the motion is determined that fixes the energy decay rate to be $t^{-1.5}$. For our problem this suggests that

$$
\begin{aligned}
& u^{\prime} \sim \frac{d \delta}{d t} \sim t^{-0.75}, \\
& \delta \sim t^{0.25} .
\end{aligned}
$$

To summarize, theoretical considerations suggest that when $A \neq 1$ the asymptotic increase of late-time RM amplitude may vary anywhere from logarithmically to linearly in time. We anticipate that at late times, when large-scale and small-scale disturbances have grown to about the same amplitude, the large scales may retain some memory of initial conditions while the small scales will be virtually indepen- dent, yielding a net small dependence of global parameters such as TMZ thickness $\delta$ on the initial configuration.

Aleshin et al., ${ }^{18}$ in a study of the growth of single-mode perturbations excited by shocks of strength $M_{s}=3.5$, were perhaps the first to observe the transition from linear to nonlinear growth in laboratory experiments. Brouillette and Sturtevant $^{19}$ conducted experiments in a $114 \mathrm{~mm}$ square shock tube on nominally plane multimode interfaces initially established by thin plastic membranes to times corresponding to $x / \delta \sim 40$, where $x=U t$. The observed growth rate induced by single incident shock waves decreased rapidly with time. The data were not sufficient to determine quantitative power-law behavior, but they showed that $0<n<1$, perhaps in the range 1/3-2/3 (see Fig. 17, Ref. 19). In recent experiments Sadot et al. ${ }^{14}$ observed the initial stages of non-linear saturated growth of single-mode perturbations to $x / \delta \sim 10$. (For the single-mode case we take $\delta=\eta-\eta_{0}$, where $\eta$ is the half-amplitude of the single-scale perturbation.) They found agreement with their interpolation formula.

Many experimental results have been reported for growth after excitation by more than one shock wave, called reshock. For example, in several experiments all carried out in the same shock tube, ${ }^{3,19,20}$ Brouillette and Sturtevant reported growth linear in time after reshock by one or more reverberations between the shock tube end wall and the interface. The rate in most cases was somewhat smaller than predicted by Eq. (2). However, experiments in a larger shock tube $^{21}$ also yielded linear growth after reshock, with growth in better agreement with Eq. (2). This agreement suggests that the observed growth after reshock is in the nonlinear saturated regime, with many spatial scales operative. Linear growth after reshock was observed at about the same time after excitation as was the highly nonlinear, decaying growth observed after only a single shock. This apparent disparity in behavior is explained by the fact that the turbulence is reenergized during reshock by shock interaction with perturbations grown by the first shock process, and any decay of the resulting growth was not observable during the available experimental time.

In this paper we describe an investigation of the growth of single-mode perturbations induced by a single incident shock wave in a large shock tube. The interface is formed by a polymeric membrane supported by a wire mesh, so smallscale perturbations, including undefined very-small perturbations due to membrane imperfections, are also present. Four different initial amplitude/wavelength combinations with $k_{0} \eta_{0}=0.31$ and 0.62 are used to investigate how the data scale with these parameters. Only one incident shock strength, $M_{s}=1.55$, is studied. The interface is followed to large time, up to 32 wavelengths $\left(k_{0} x=200\right.$, or $\left.x / \delta=20\right)$ from the initial location, and the data are analyzed for the power-law dependence of TMZ thickness on time. We determine the power law which best collapses the four amplitude/ wavelength data sets to one curve, and we examine any residual dependence of the fit on initial conditions. A study is made of the effect of a supporting wire mesh on the growth rate. Any examination of the asymptotic late-time behavior of the RM instability is necessarily greatly separated in both spatial and temporal scale from the initial small-amplitude 


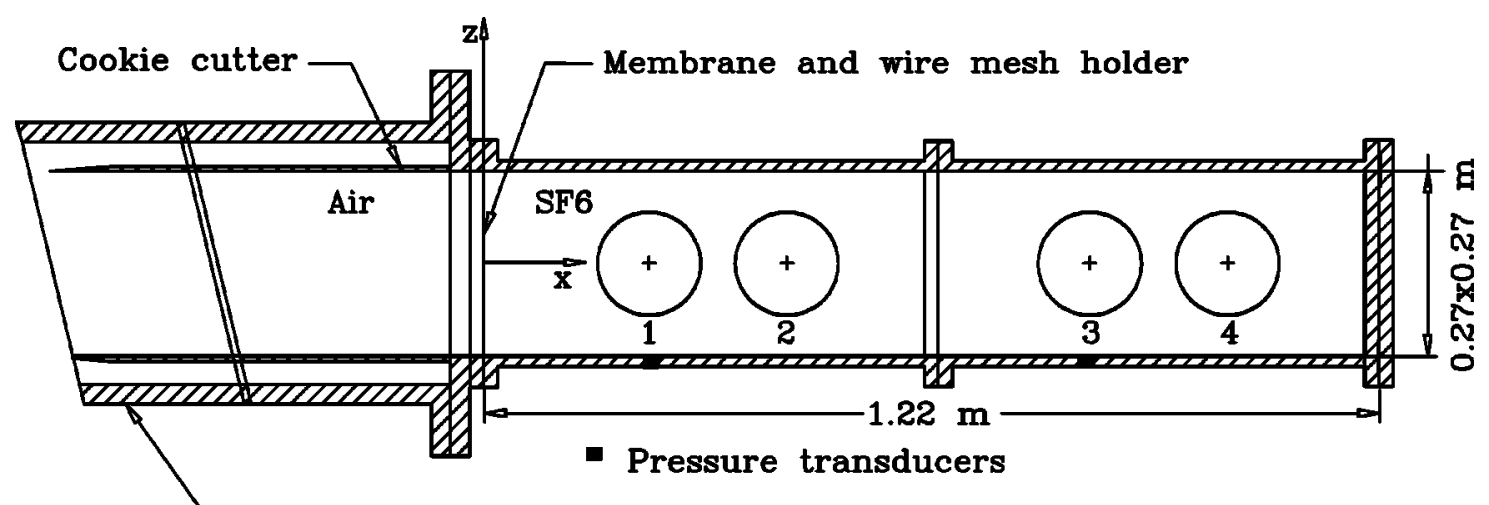

\subsection{3 dia Shock Tube}

FIG. 1. Schematic drawing of the test section showing observation window placement.

linear regime. Thus it is difficult in any single experiment to access both regimes, and, as in most previous shock tube studies of the RM instability, this has not been attempted in the present experiments. Linear growth at the Atwood and Mach numbers of these experiments is undetectable with the present apparatus.

\section{EXPERIMENTS}

The experiments were carried out in the GALCIT $17 \mathrm{in.}$ diam shock tube. ${ }^{21,22}$ A square $267 \times 267 \mathrm{~mm}(10.5 \times 10.5$ in.) test section $1.22 \mathrm{~m}$ long was adapted to the end of the shock tube by insertion of a "cookie cutter" into the circular cross section (Fig. 1). Viewing windows $152 \mathrm{~mm}$ in diameter could be centered at positions 226, 429, 836, and $1039 \mathrm{~mm}$ downstream of the initial location of the interface. The run conditions and the wavelengths and initial amplitudes of the four single-scale configurations studied are given in Table I. A thin $1.5 \mu \mathrm{m}$ thick nitrocellulose membrane supported by an array of thin horizontal wires formed the initial quasicosinusoidal profile of the interface. Because of the large crosssectional size of the test section, wires spanning the test section were necessary to define the interface shape. The wire array consisted of pairs of parallel $0.23 \mathrm{~mm}$ diam wires positioned approximately $1 \mathrm{~mm}$ apart on either side of the membrane. The number of wires was chosen in a tradeoff between the accuracy to which the membrane shape would approximate a cosine wave and the magnitude of the flow disturbance caused by the wires. Twenty-six equally-spaced pairs of wires were used for Frames 1-3, while 53 pairs spaced in a 3.3-8.2-3.3 $\mathrm{mm}$ repeating sequence to optimize the definition of curvature near the crests and troughs were used for Frame 4. Figure 2 indicates schematically the inter- face configuration as formed near the origin $(x, z=0)$ by Frames 2 and 4. After shock acceleration, the membrane was cut into ribbons by the downstream set of wires.

The nominal shape of the interfaces is described by

$$
\eta(z)=-\eta_{0} \cos k_{0} z ; \quad-133.4 \leqslant z \leqslant 133.4 \mathrm{~mm},
$$

where the coordinates are defined in Figs. 1 and 2, $\eta$ increases in the direction of increasing $x$, and $k_{0}$ and $\eta_{0}$ are given in Table I. The sign on $\eta_{0}$ indicates that on the centerline of the tube all membranes initially formed a crest pointed upstream relative to the mean position of the interface, $x=0$. An exception was Run 014 (Fig. 3), in which $\eta_{0}<0$. The cosinusoidal shape is maintained accurately around the perimeter of the shock tube by the machined frame. However, near the center of the shock tube (on the vertical axis of symmetry of the wire mesh), the membrane was stretched against the upstream wires, as described by Vetter and Sturtevant. ${ }^{21}$ This introduced higher harmonics into the nominally cosinusoidal shape. For example, the amplitude of the second harmonic, expressed as a percentage of the first harmonic, was $3.7 \%$ for Frames 1 and 2, 1.2\% for Frame 3, and $0.9 \%$ for Frame 4. Furthermore, imperfections in the nitrocellulose membrane introduced additional undefined very-small-scale perturbations.

The run procedure and instrumentation described by Vetter and Sturtevant ${ }^{21}$ were used in these experiments. The initial test-section pressure was $23 \mathrm{kPa}$. A single sparkschlieren photograph of the interface or a series of images from a high speed motion picture taken at $40000 \mathrm{fps}$ was obtained during each run. The time history was constructed from records taken at the four different window positions.

TABLE I. Experimental conditions. Air $/ \mathrm{SF}_{6}$ interface, $M_{s}=1.55, U_{I}=174 \mathrm{~m} / \mathrm{s}, A=0.67, A^{\prime}=0.74$.

\begin{tabular}{ccccccccccc}
\hline \hline Frame & $\begin{array}{c}\lambda_{0} \\
(\mathrm{~mm})\end{array}$ & $\begin{array}{c}k_{0} \\
\left(\mathrm{~mm}^{-1}\right)\end{array}$ & $\begin{array}{c}\eta_{0} \\
(\mathrm{~mm})\end{array}$ & $k_{0} \eta_{0}$ & $\begin{array}{c}x_{\min } \\
(\mathrm{mm})\end{array}$ & $\begin{array}{c}x_{\max } \\
(\mathrm{mm})\end{array}$ & $k_{0} x_{\min }$ & $k_{0} x_{\max }$ & $n$ & Symbol \\
\hline 1 & 59.3 & 0.106 & 2.97 & 0.31 & 175 & 874 & 18.6 & 92.6 & 0.23 & $\triangle$ \\
2 & 59.3 & 0.106 & 5.91 & 0.62 & 188 & 835 & 19.9 & 88.5 & 0.21 & $\square$ \\
3 & 106.7 & 0.059 & 5.33 & 0.31 & 210 & 1020 & 12.4 & 60.2 & 0.34 & $\diamond$ \\
4 & 29.6 & 0.212 & 1.46 & 0.31 & 242 & 955 & 51.3 & 202.5 & 0.20 & $\bigcirc$ \\
\hline \hline
\end{tabular}




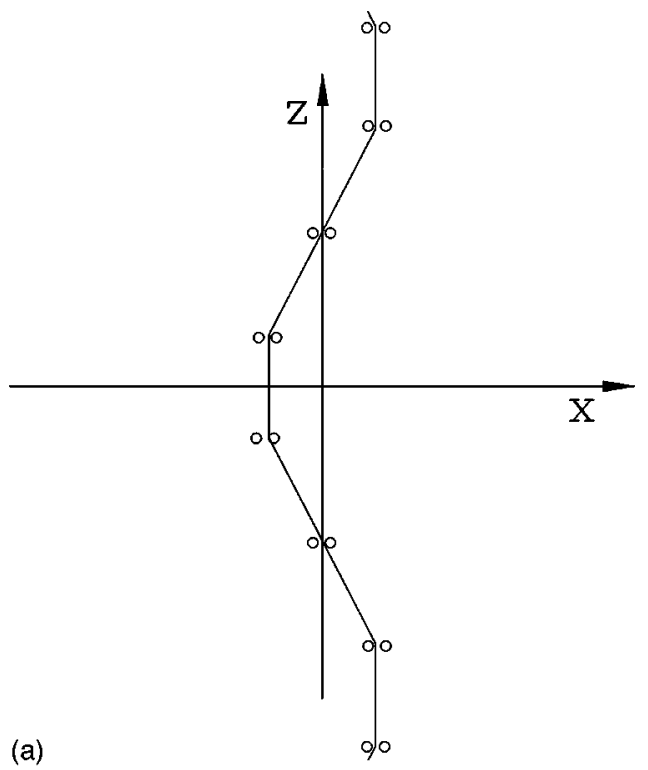

(b)

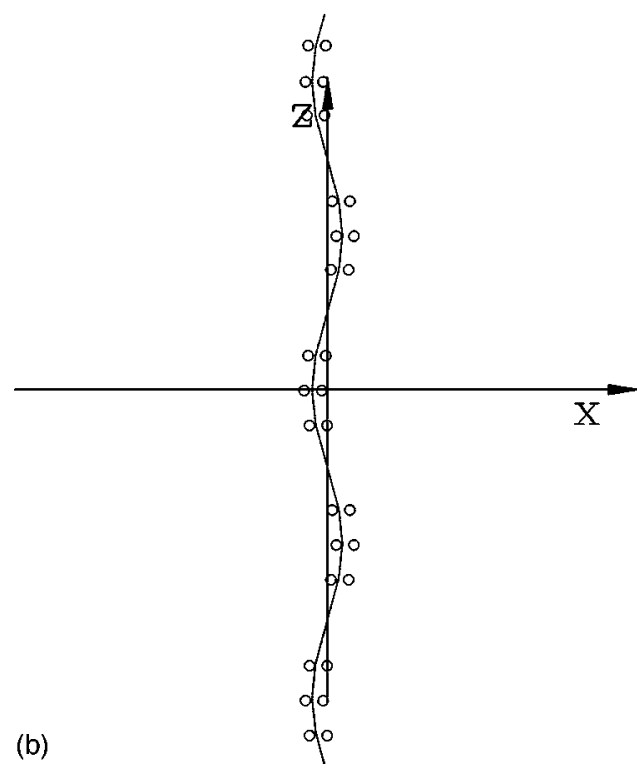

FIG. 2. Schematic diagram of one wavelength of the membrane near $x$ $=0, z=0$, showing the arrangement of the wire mesh. (a) Frame 2, (b) Frame 4. Scale: Distance from the origin to the end of each arrow $=30 \mathrm{~mm}$.

The limits of the streamwise positions downstream of the initial location of the interface at which measurements were obtained are given in Table I.

Helium was used as the driver gas in the shock tube. For the present run conditions the expansion wave from the driver section overtook the incident shock in the test section, so behind the shock the pressure decreased at a measured rate of $1.5 \mathrm{kPa} / \mathrm{ms}$ or $0.62 \%$ per wavelength (of Frames 1 and 2) traveled. Thus the impulsive acceleration by the shock was followed by a small constant deceleration, superposing a small stabilizing RT effect on the RM growth. This effect was also present in the experiments of Ref. 21. In view of its small amplitude, it is ignored in our presentation of the present results, but it could be accounted for, for example, in numerical simulations. We note that postshock deceleration and reshock always occur in convergent geometries, as with

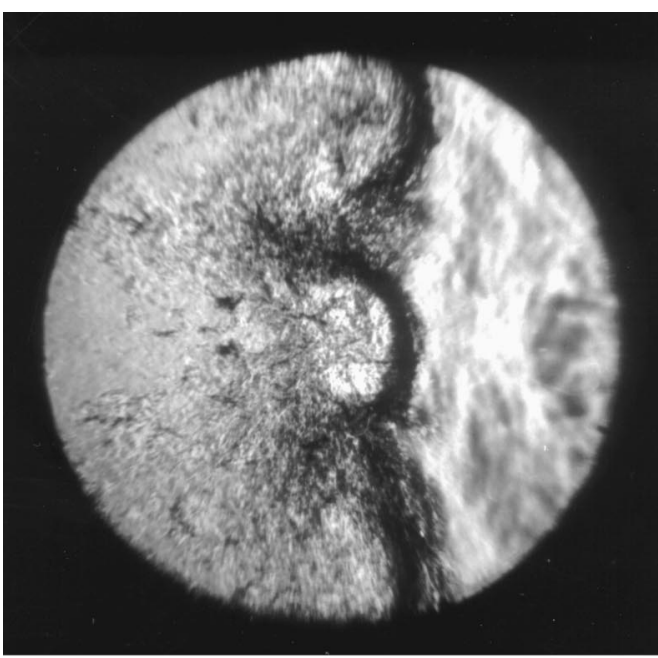

(a)

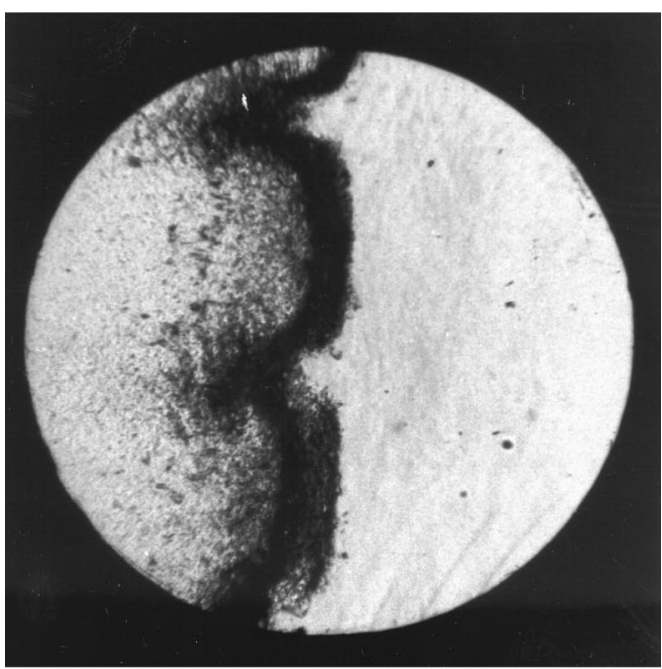

(b)

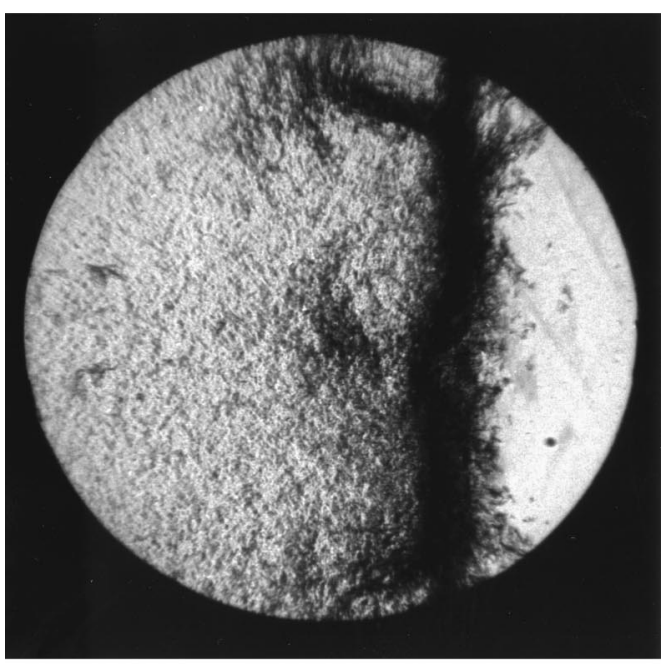

(c)

FIG. 3. Single schlieren images, each taken during a separate run, of the Frame 1 mixing layer. $\lambda_{0}=59.1 \mathrm{~mm} . \eta_{0}= \pm 2.97 \mathrm{~mm}, k_{0} \eta_{0}=0.31$. (a) $x$ $=219 \mathrm{~mm}(t=1.47 \mathrm{~ms}), M_{s}=1.57$, Run $014\left(\eta_{0}<0\right)$; (b) $x=405 \mathrm{~mm}(t$ $=2.54 \mathrm{~ms}), M_{s}=1.51$, Run 031; (c) $x=848 \mathrm{~mm}(t=5.44 \mathrm{~ms}), M_{s}=1.55$, Run 034. 


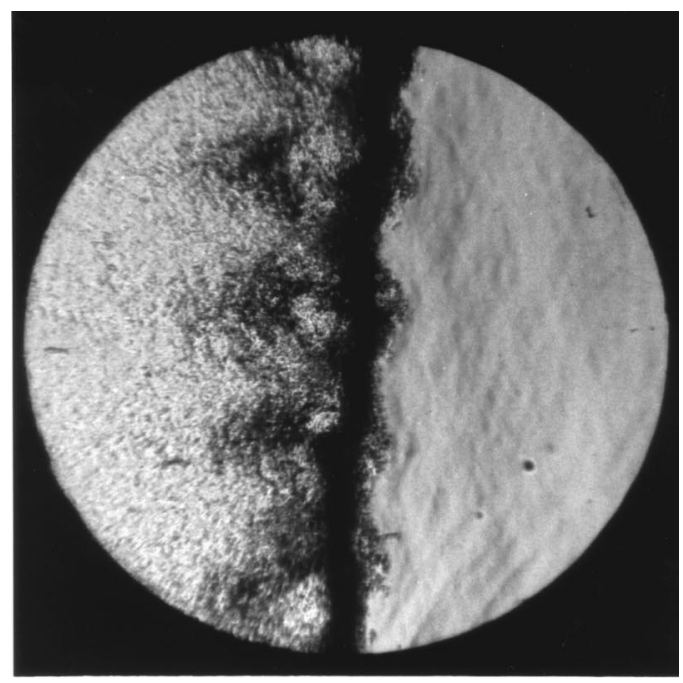

(a)

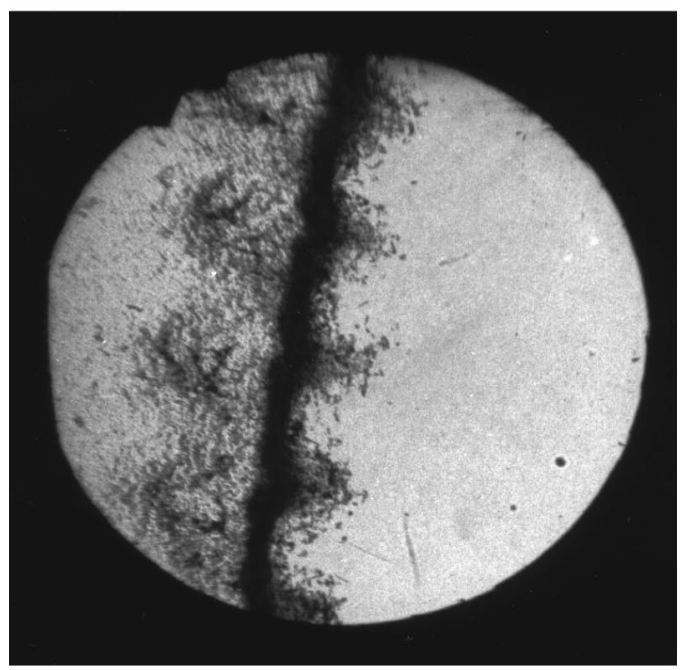

(b)

FIG. 4. Schlieren images of the Frame 4 mixing layer. $\lambda_{0}=29.6 \mathrm{~mm}, \eta_{0}$ $=1.46 \mathrm{~mm}, k_{0} \eta_{0}=0.31$. (a) $x=417 \mathrm{~mm}(t=2.61 \mathrm{~ms}), M_{s}=1.52$, Run 032; (b) $x=815 \mathrm{~mm}(t=5.36 \mathrm{~ms}), M_{s}=1.50$, Run 027 .

spherically imploding shocks onto spherical interfaces. Therefore, numerical simulations of the RM instability will routinely include a superposition of the RM, RT, and reshock effects. A numerical study of the effect of small decelerations in the present experimental configuration would be useful.

\section{RESULTS}

Figure 3 shows a sequence of three spark schlieren photographs of Frame 1, and Fig. 4 shows photographs for Frame 4, with one half the wavelength and one half the initial amplitude of Frame 1. Figure 5 shows examples of images from Frames 2 and 3. Air is on the left and sulfur hexafluoride $\left(\mathrm{SF}_{6}\right)$ is on the right. The interface is imaged dark because of a combination of the schlieren effect and the scattering of light from the field of view by membrane fragments. The interface in all pictures is clearly in the nonlinear regime, with bubbles "rising" into the heavy $\mathrm{SF}_{6}$ on the

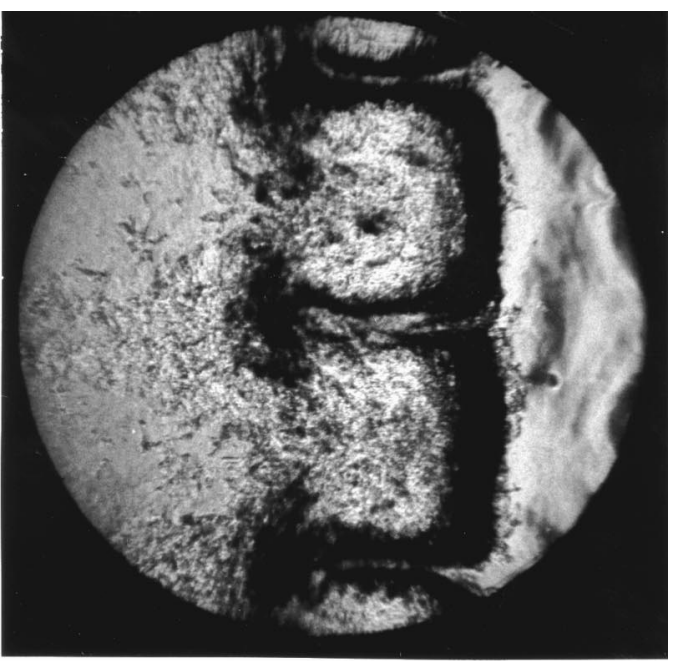

(a)

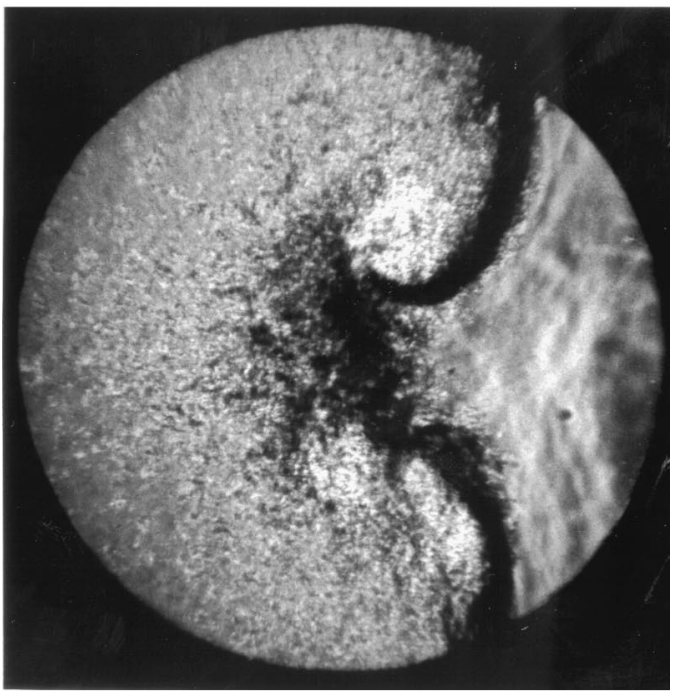

(b)

FIG. 5. Schlieren images of (a) Frame 2 and (b) Frame 3. (a) $\lambda_{0}=59.1 \mathrm{~mm}$, $\eta_{0}=5.91 \mathrm{~mm}, k_{0} \eta_{0}=0.62, x=231 \mathrm{~mm}(t=1.51 \mathrm{~ms}), M_{s}=1.58$, Run SK23; (b) $\lambda_{0}=106.7 \mathrm{~mm}, \eta_{0}=5.33 \mathrm{~mm}, k_{0} \eta_{0}=0.31, x=233 \mathrm{~mm}(t$ $=1.52 \mathrm{~ms}), M_{s}=1.56$, Run SK24.

right and spikes "falling" into the air. At large times the bubbles are observed to flatten, and nascent spikes appear protruding into the $\mathrm{SF}_{6}$ just opposite the original $\mathrm{RM}$ spikes. The cause of this development is not known, but it may be due to the cumulative effect of the small stabilizing RT deceleration described above.

A dimensional plot of the results of measurements of the half thickness $\delta=\eta-\eta_{0}$ at different interface positions $x$, for all of the experiments reported here is given in Fig. 6. $x$ is proportional to the time $t$ after shock excitation, $x=U t$. The raw data used in all of the plots of this paper are available in numerical form at http://www.galcit.caltech.edu/ $\sim$ brad/hyper/rundata/.

A trend of growth rate with initial amplitude and wavelength is evident in the figure; the smallest initial amplitudes have slightly smaller thickness, and the largest wavelength is the thickest. 


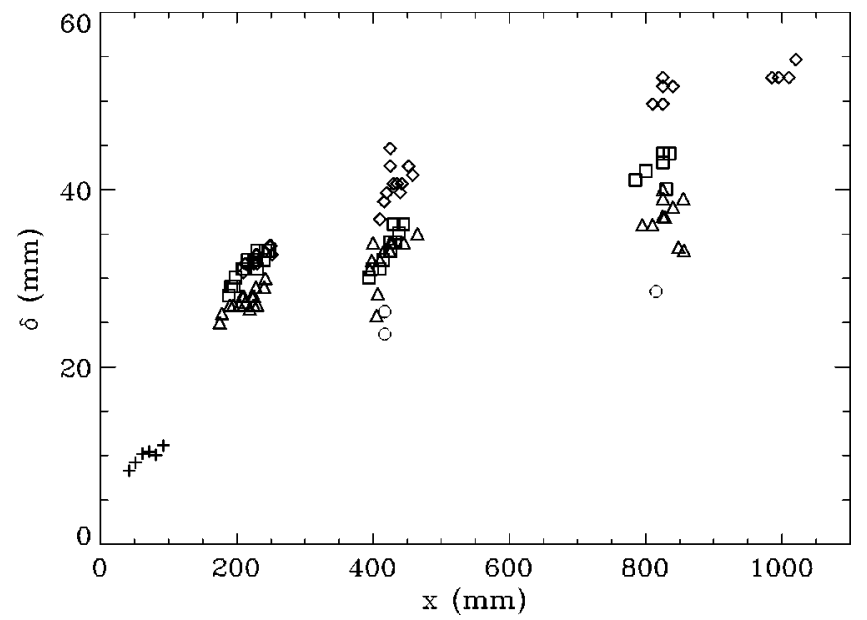

FIG. 6. Plot of TMZ thickness vs position of the interface for different wavelengths and initial amplitudes. Symbols defined in Table I. Smallest initial amplitudes $(\bigcirc$ and $\triangle$ ) show the smallest growth, while the largest wavelength $(\diamond)$ grows the fastest. +, late-time data of Sadot et al. (Ref. 15) for $\lambda_{0}=16 \mathrm{~mm}$.

\section{A. Scaling}

In presentations of experimental and theoretical results, the amplitude $\delta=\eta-\eta_{0}$ has sometimes been scaled with the initial amplitude $\eta_{0}$. However, Fig. 6 shows that, though Frame $1(\triangle)$ has one half the initial amplitude of Frame 2 ( $\square$ ) and the same wavelength, its amplitude is approximately the same, except for $x>800 \mathrm{~mm}$, where it is somewhat smaller. Consequently, if the thickness were to be normalized by $\eta_{0}$, the triangles would be moved far above the squares and the scatter would be increased. We conclude that this is not the appropriate scaling for the present data. Frame $3(\diamond)$ has the same initial amplitude as Frame $2(\square)$, but twice the wavelength, and its growth is substantially larger than both Frame 1 and 2. Normalization of the thickness by the wavenumber $k_{0}$ reduces this discrepancy. Thus, we select the normalization shown in Fig. 7, which largely collapses the data. Least squares power law fits, $k_{0} \delta \propto\left(k_{0} x\right)^{n}$, to each of the four initial amplitude and wavelength configurations studied are shown in Fig. 7(a), and the resulting values of $n$ are given in Table I. A composite least squares best fit to all of the data,

$$
k_{0} \delta=0.96\left(k_{0} x\right)^{0.33}
$$

is shown in Fig. 7(b).

A further study was made to determine which power law and combination of parameters optimally reduces the scatter of the data, and thereby minimizes the apparent dependence on initial conditions. It was found that the scatter of the data in Fig. 7 can be significantly reduced by the dimensional scaling

$$
\delta=2.43\left(\lambda_{0} x\right)^{0.26},
$$

where all lengths are expressed in $\mathrm{mm}$. The result is shown in Fig. 8.
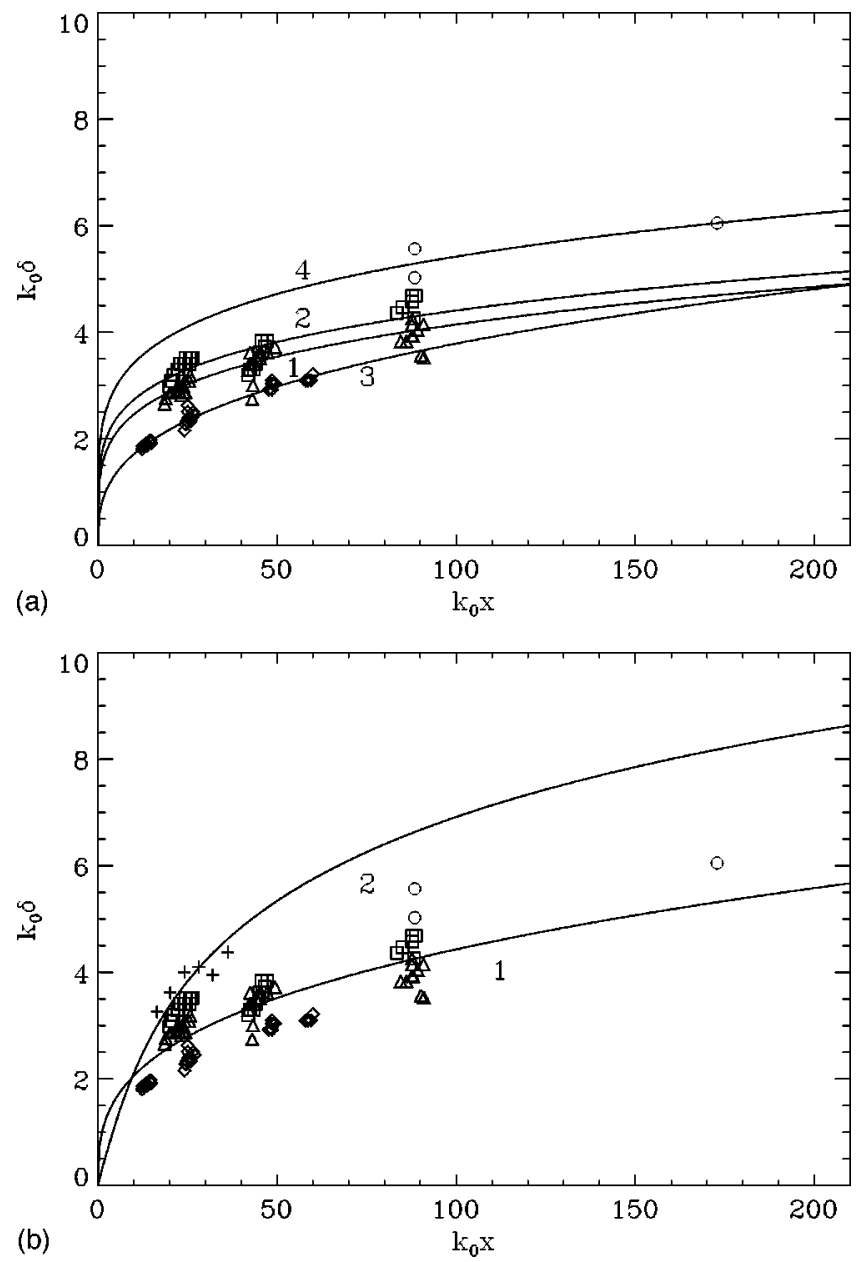

FIG. 7. (a) Power law fits, $k_{0} \delta \propto\left(k_{0} x\right)^{n}$, to normalized data for the four different interfaces. The values of $n$ are given in Table I. Curve numbers indicate interface (see Table I). (b) 1 , Best fit power law function, $k_{0} \delta$ $=0.96\left(k_{0} x\right)^{0.33}$, to data for all four interfaces. Symbols listed in Table I. 2, Power series fit, Eq. (1) of Sadot et al. (Ref. 15), for $k_{0} \eta_{0}=0.42, A$ $=0.72 .+$, late-time data of Sadot et al. (Ref. 15) for $\lambda_{0}=16 \mathrm{~mm}$.

\section{B. Effect of membrane and wires on mixing}

The effect of using a thin plastic membrane to initially form the interface for studies of the RM instability is that it tends to suppress mixing by isolating the two test gases from each other and by retarding motions, owing to its inertia and the viscous no-slip condition. On the other hand, the mesh of parallel wires that initially supports the membrane enhances the mixing for two reasons. First, it slices the membrane into ribbons and so tends to negate the above-mentioned suppression. Second, the wires generate a turbulent field upstream of the interface. The turbulent wakes of the wires near the interface enhance mixing. Furthermore, membrane fragments clinging to the wire mesh may increase the drag of the mesh and consequently the turbulence level in the neighborhood of the interface. The more wires that are used, the larger is the drag of the mesh and the mesh-induced mixing. Thus, we have chosen to use as few wires as possible to support the membrane.

A study was made of the effect of the wires by removing some of them in the upper third of the frame. One wire at the edge of a trough (that is, pointing toward $\mathrm{SF}_{6}$ ) and two wires 


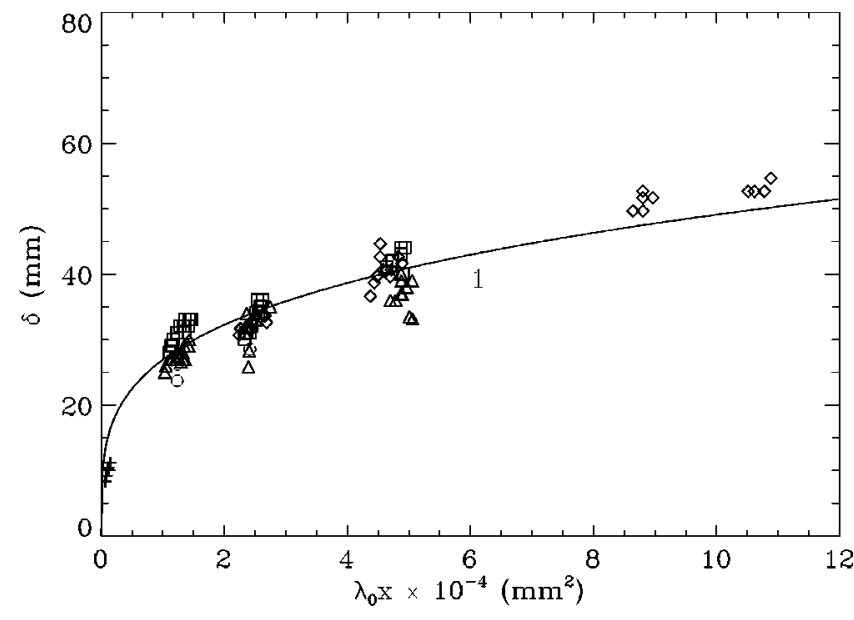

FIG. 8. Correlation which yields minimum scatter of present data about a power-law fit. Symbols listed in Table I. $1, \delta=2.43\left(\lambda_{0} x\right)^{0.26} ; \delta, \lambda_{0}$ and $x$ in mm. + , late-time data of Sadot et al. (Ref. 15) for $\lambda_{0}=16 \mathrm{~mm}$.

at the nearest crest above (pointing toward air) were removed. This had the secondary effect of introducing a triangular waveform for one-half wavelength near the center of the shock tube and an amplitude reduction of about $10 \%$, averaged over the shock tube cross section (the frame maintained the shape truly sinusoidal along the edges). Since in these experiments the observed dependence on initial amplitude is small, the small decrease of amplitude probably had little effect on the growth rate. Figure 9 shows a schlieren image with lines superposed to indicate the region where the wires were removed, and to help visualize the growth decrease of about $20 \%$. We conclude that the wire mesh used had the effect of a modest increase in growth rate.

\section{DISCUSSION}

Measurements of the amplitude of single-scale perturbations on an interface between air and $\mathrm{SF}_{6}$ accelerated by an

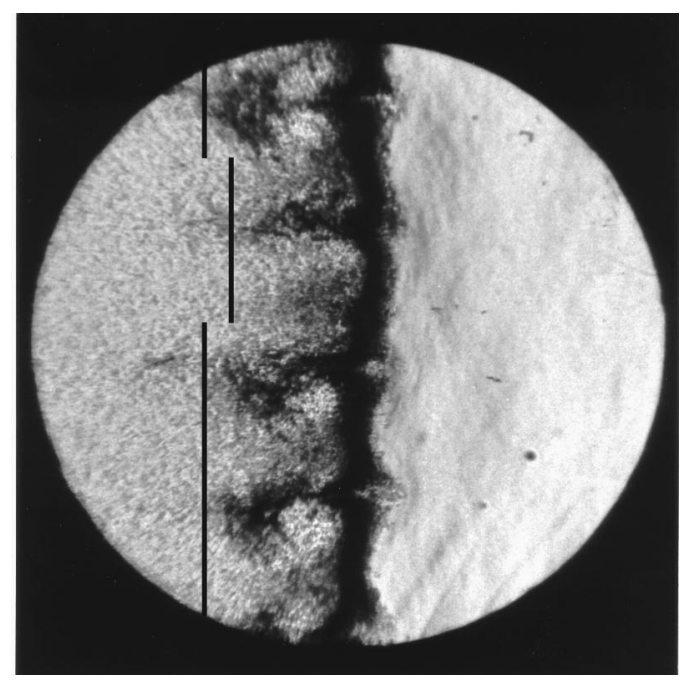

FIG. 9. Schlieren image of the Frame 4 mixing layer at window position \#2 to determine the effect of the support mesh on mixing. Region where wires were removed, and an estimate of the reduction of growth indicated by black lines. $x=429 \mathrm{~mm}(t=2.59 \mathrm{~ms}), M_{s}=1.54$, Run 033 .
$M_{s}=1.55$ shock wave show that at late times the growth rate decreases rapidly with time, $d \delta / d t \propto t^{-p}$, where $0.67 \lesssim p$ $\lesssim 0.74$. Our scaling results do not give any information about the dependence on shock strength and Atwood number, because only one value of each was used in these experiments. And only the RT unstable fast-slow configuration was studied. Furthermore, our study of the dependence on initial conditions, Eq. (8), relies on only three different amplitudes and wavelengths. The observed decreasing growth rate with increasing time is accompanied by flattening of the bubbles on the nonlinearly deformed interface.

As can be seen from the schlieren photographs, the "'growth", which we measure derives from both the increase of single-scale perturbations on a large spatial scale, owing to baroclinically-generated interfacial vorticity, and a diffuse thickening of the interfacial region, presumably by turbulent diffusion at small scale. In single-scale experiments studying thin interfaces not initially diffusively smoothed, small-scale imperfections on the interface always ensure that a superposition of both single-scale and multiscale perturbations are present from the outset. As previously discussed, it is expected that large-time self-similar behavior will occur when the scale of the small-scale structure reaches that of the single-scale, ${ }^{6}$ and the influence of the initial conditions decreases. It is clear that the small-scale diffusive growth, which is of the same magnitude as that observed in earlier purely multiscale experiments in this same shock tube, ${ }^{21}$ is a major, if not the major, contributor to the growth recorded in these experiments. The images of the density field reflect the entire history of distortion and mixing at the interface, beginning with shock acceleration. Even though a single large scale persists visually, it may be that the small-scale turbulent diffusion is dynamically dominant at these large times.

We note that Eq. (8) agrees to well within the experimental error with the power law given by the Saffman invariant in the Huang-Leonard similarity law, Eq. (5). This suggests that the invariance of the second moment of the velocity correlation proposed by $\mathrm{Saffman}^{17}$ applies to latetime RM mixing. At the latest time in our experiments the Reynolds number Eq. (4) based on the mean of the viscosities of air and $\mathrm{SF}_{6}$ is about 15000 , so the Reynolds number based on the Taylor microscale $\lambda$ is about $\operatorname{Re}_{\lambda} \sim 100-300$. The values $p=-0.6, n=0.4$ suggested by an alternative argument of Saffman ${ }^{23}$ and obtained numerically by Alon et al. $^{11}$ and Pham and Meiron ${ }^{15}$ fall slightly outside of the range obtained by the fits to our data derived in Sec. III A.

In Eq. (8) all quantities have units of length. The form $\delta \sim\left(\lambda_{0} x\right)^{n}$ implies that there is an asymptotic flowgenerated length scale, say $l^{\prime}$, during the late-time selfsimilar decay such that

$$
\delta \sim l^{\prime m}\left(\lambda_{0} x\right)^{n}
$$

where, by dimensional reasoning, $m=1-2 n$. Implicit in the relation Eq. (9) is the assumption that $l^{\prime}$ is a constant (i.e., varying in time much more slowly than $\delta$ ). If, as might be expected, dissipation during the late stages of decay is dominated by viscosity, then $l^{\prime}$ should scale with the viscous 
length, $l^{\prime} \sim \nu / u^{\prime}$, where $u^{\prime}$ is, say, the magnitude of the turbulent velocity fluctuation at time $t$, again taken to be slowly varying. Then

$$
\delta \sim\left(\frac{\nu}{u^{\prime}}\right)^{m}\left(\lambda_{0} x\right)^{n} .
$$

Taking from Eq. (8) that $n \approx 1 / 4$, we conclude that approximately $m=1 / 2$; that is, $\delta \sim \nu^{1 / 2}$. The parabolic behavior implied by this result might be taken to suggest that at late times viscosity acts at effectively high Reynolds number, i.e., is confined to thin layers. However, caution should be used in interpreting this result, because implications from the data are insufficiently resolved to warrant such general conclusions.

Although the $t^{-0.74}$ approach of the growth rate toward zero observed in these experiments is rather rapid, it is slower than the $t^{-1}$ behavior proposed in some earlier work, ${ }^{12,14}$ and when plotted as $\delta(t)$ [Fig. 7(b)], it is easily distinguishable from that behavior over the time scales of our experiments. In fact, it is likely that the late-time decay observed in the present experiments is somewhat more rapid ( $p$ larger, $n$ smaller) than that of the RM instability of an ideal, discontinuous, membrane-less sinusoidal interface because of the (i) small stabilizing RT deceleration that follows the impulsive RM acceleration in our shock tube, (ii) damping effects of membrane fragments, and (iii) possible enhanced growth at early time. Two mechanisms which can induce growth primarily at early times are the small-scale turbulence initially deposited at the interface by the supporting wire mesh and the effect of small-scale perturbations on the interface due to membrane imperfections, both of which grow more rapidly than large scales [see Eq. (3)]. Near the center of the shock tube the membrane does not initially have a smooth sinusoidal shape, but is stretched in straight-line segments across the wires (Fig. 2). The resulting sharp corners also induce small-scale motions (see Sec. II). On the other hand, these experiments are conducted in a large shock tube, so thinning of the interface due to the sink effect of the nonsteady boundary layers, which is important in smaller shock tubes and yields an apparently slower growth rate, is avoided. We conclude that the measurements reported here yield a lower bound to the power $n$ with which the thickness of RM interfaces grow at large time.

The large-time asymptotics of the RM instability deserves further study in laboratory experiments. Dependence on shock strength and the magnitude and sign of the Atwood number should be examined. Further tests of the effects of wire mesh density and membrane thickness are warranted.

\section{ACKNOWLEDGMENT}

This work was supported by Contract B160540, Lawrence Livermore National Laboratory.
${ }^{1}$ R. D. Richtmyer, "Taylor instability in shock acceleration of compressible fluids," Commun. Pure Appl. Math. 13, 297-319 (1960).

${ }^{2}$ E. E. Meshkov, "Instability of the interface of two gases accelerated by a shock wave," Soviet Fluid Dynamics 4, 101-104 (1969).

${ }^{3}$ M. Brouillette and B. Sturtevant, "Growth induced by multiple shock waves normally incident on plane gaseous interfaces," Physica D 37, 248-263 (1989).

${ }^{4}$ R. Briedenthal, AIAA J. 17, 310-311 (1979).

${ }^{5}$ Bernal et al., in Turbulent Shear Flows 2, edited by L. J. S. Bradbury et al. (Springer-Verlag, Berlin, 1980), pp. 81-86.

${ }^{6} \mathrm{~S}$. W. Haan, “'Onset of nonlinear saturation for Rayleigh-Taylor growth in the presence of a full spectrum of modes," Phys. Rev. A 39, 58125825 (1989).

${ }^{7}$ G. I. Barenblatt "'Self-similar turbulence propagation from an instantaneous plane source," in Nonlinear Dynamics and Turbulence, edited by G. I. Barenblatt, G. Iooss, and D. D. Joseph (Pitman, Boston, 1983), pp. $48-60$.

${ }^{8}$ K. O. Mikaelian, “Turbulent mixing generated by Rayleigh-Taylor and Richtmyer-Meshkov instabilities," Physica D 36, 343-357 (1989).

${ }^{9}$ K. I. Read, "Experimental investigation of turbulent mixing in RayleighTaylor instability,' Physica D 12, 45 (1984).

${ }^{10}$ U. Alon, J. Hecht, D. Mukamel, and D. Shvarts, "Scale invariant mixing rates of hydrodynamically unstable interfaces," Phys. Rev. Lett. 72, 2867-2870 (1994).

${ }^{11}$ U. Alon, J. Hecht, D. Ofer, and D. Shvarts, "Power laws and similarity of Rayleigh-Taylor and Richtmyer-Meshkov mixing fronts at all density ratios,"' Phys. Rev. Lett. 74, 534-537 (1995).

${ }^{12} \mathrm{Q}$. Zhang and S. Sohn, "Nonlinear theory of unstable fluid mixing driven by shock wave," Phys. Fluids 9, 1106-1124 (1997).

${ }^{13}$ R. F. Benjamin, "Experimental observations of shock stability and shockinduced turbulence," in Advances in Compressible Turbulent Mixing, edited by W. P. Dannevik, A. C. Buckingham, and C. E. Leith, Conf8810234, 341-348 (Nat. Tech. Inf. Service, Virginia, 1992).

${ }^{14}$ O. Sadot, L. Erez, U. Alon, D. Oron, L. A. Levin, G. Erez, G. Ben-Dor, and D. Shvarts, "Study of nonlinear evolution of single-mode and twobubble interaction under Richtmyer-Meshkov instability," Phys. Rev. Lett. 80, 1654 (1998).

${ }^{15}$ T. Pham and D. I. Meiron, "A numerical study of Richtmyer-Meshkov instability in continuously stratified fluids," Phys. Fluids 5, 344-368 (1993).

${ }^{16}$ M.-J. Huang and A. Leonard, "Power-law decay of homogeneous turbulence at low Reynolds numbers," Phys. Fluids 6, 3765-3775 (1994).

${ }^{17} \mathrm{P}$. G. Saffman, "The large-scale structure of homogeneous turbulence,", J. Fluid Mech. 27, 581-593 (1967).

${ }^{18}$ A. N. Aleshin, E. G. Gamalii, S. G. Zaitsev, E. V. Lazareva, I. G. Lebo, and V. B. Rozanov, "Nonlinear and transitional stages in the onset of the Richtmyer-Meshkov instability," Sov. Tech. Phys. Lett. 14, 466-468 (1988).

${ }^{19}$ M. Brouillette and B. Sturtevant, "Experiments on the RichtmyerMeshkov instability; small scale perturbations on a plane interface," Phys. Fluids 5, 916-930 (1993).

${ }^{20} \mathrm{M}$. Brouillette and B. Sturtevant, "Experiments on the RichtmyerMeshkov instability; single scale perturbations on a continuous interface," J. Fluid Mech. 263, 271-292 (1994).

${ }^{21} \mathrm{M}$. Vetter and B. Sturtevant, "Experiments on the Richtmyer-Meshkov instability of an Air/SF6 interface," Shock Waves 4, 247-252 (1995).

${ }^{22}$ H. W. Liepmann, A. Roshko, D. Coles, and B. Sturtevant, " A 17 in. diam shock tube for studies in rarefied gas dynamics," Rev. Sci. Instrum. 33, 625-631 (1962).

${ }^{23}$ P. G. Saffman, "Note on the decay of homogeneous turbulence," Phys. Fluids 10, 1349 (1967). 